Published in final edited form as:

Int J Cardiol. 2015 April 15; 185: 138-140. doi:10.1016/j.ijcard.2015.03.038.

\title{
FITBIT®: AN ACCURATE AND RELIABLE DEVICE FOR WIRELESS PHYSICAL ACTIVITY TRACKING
}

\author{
Keith M. Diaz ${ }^{1}$, David J. Krupka ${ }^{1}$, Melinda J Chang ${ }^{1}$, James Peacock ${ }^{1}$, Yao Ma ${ }^{2}$, Jeff \\ Goldsmith $^{2}$, Joseph E. Schwartz ${ }^{1}$, and Karina W. Davidson ${ }^{1}$ \\ ${ }^{1}$ Center for Behavioral Cardiovascular Health, Department of Medicine, Columbia University \\ Medical Center, New York, NY. This author takes responsibility for all aspects of the reliability and \\ freedom from bias of the data presented and their discussed interpretation \\ 2Department of Biostatistics, Mailman School of Public Health, Columbia University, New York, \\ NY. This author takes responsibility for all aspects of the reliability and freedom from bias of the \\ data presented and their discussed interpretation
}

\section{Keywords}

Physical activity; validation; activity monitoring; accelerometer; walking; Fitbit

\begin{abstract}
Although physicians recognize the importance of physical activity in the prevention and maintenance of chronic diseases, ${ }^{1}$ few incorporate physical activity counseling into routine clinic visits. ${ }^{2}$ One major barrier has been the paucity of means to objectively assess patient's long-term physical activity patterns. ${ }^{3}$ We investigated if the Fitbit ${ }^{\circledR}$, one of the most common wireless physical activity trackers in the consumer market, met reasonable validity and reliability standards such that they could be used by primary care physicians to monitor their patient's physical activity objectively between clinic visits.
\end{abstract}

Twenty-three healthy adult participants (10 males; age range:20-54 years; body mass index range:19.6-29.9 kg/m²) completed a four-stage treadmill exercise protocol consisting of walking at slow $(1.9 \mathrm{mph})$, moderate $(3.0 \mathrm{mph})$, and brisk $(4.0 \mathrm{mph})$ paces; and jogging (5.2 $\mathrm{mph})$. Each stage was 6 minutes in duration. Participants were fitted with three hip-based Fitbit One ${ }^{\circledR}$ (two on right, one on left hip) and two wrist-based Fitbit Flex ${ }^{\circledR}$ (one on right and left wrist) devices. Minute-by-minute estimated-energy expenditure and step counts from Fitbit ${ }^{\circledR}$ devices were extracted from the manufacturer's website using Fitabase (Small Steps Labs). Energy expenditure was assessed by gas exchange indirect calorimetry (Ultima

(C) 2015 Published by Elsevier Ireland Ltd.

Author Correspondence: Keith M. Diaz, Columbia University Medical Center, 622 West $168^{\text {th }}$ Street, PH 9-319, New York, NY 10032, Tel\#: 212-304-5231, Fax\#: 212-305-3172, kd2442@ columbia.edu.

Conflicts of Interest: None

Publisher's Disclaimer: This is a PDF file of an unedited manuscript that has been accepted for publication. As a service to our customers we are providing this early version of the manuscript. The manuscript will undergo copyediting, typesetting, and review of the resulting proof before it is published in its final citable form. Please note that during the production process errors may be discovered which could affect the content, and all legal disclaimers that apply to the journal pertain. 
CPX, MedGraphics) and aggregated to minute-by-minute epochs. Steps were assessed by manual counting of a video recording in minute epochs.

Estimated-step counts and energy expenditure from Fitbit ${ }^{\circledR}$ devices were compared to criterion measures of observed step counts and energy expenditure from indirect calorimetry, respectively. All minutes from each stage were analyzed. This study adhered to the guidelines set forth by the Declaration of Helsinki and was approved by the institutional review board at the participating institution. All participants provided informed consent.

Estimated step counts from the hip- and wrist-based Fitbit ${ }^{\circledR}$ devices strongly correlated with observed steps counts (Figure, panels A, B). Across phases, the within-participant correlation of Fitbit-estimated step counts to observed step counts was $0.97-0.99$ for all hip devices and 0.77-0.85 for wrist devices. The mean difference of Fitbit-estimated step counts from observed step counts ranged from -3.1 to -0.3 steps and -26.3 steps to -2.9 steps for the hip- and wrist-based devices, respectively (Table, top panel). The greatest differences were seen in the wrist-based Fitbit ${ }^{\circledR}$ during slow, moderate, and brisk walking as step counts were underestimated during these stages by $16.3 \%, 10.6 \%$, and $11.3 \%$, respectively (right wrist).

Hip- and wrist-based Fitbit ${ }^{\circledR}$ estimates of energy expenditure strongly correlated with measured energy expenditure (Figure, panels C, D). Across phases, the within-participant correlation of Fitbit-estimated energy expenditure to measured energy expenditure was $0.86-0.87$ for all hip devices and 0.88 for both wrist devices. The mean difference of Fitbitestimated energy expenditure from measured energy expenditure ranged from -0.8 to 0.4 kcals and -0.2 to 2.6 kcals for the hip- and wrist-based Fitbit ${ }^{\circledR}$ devices, respectively (Table, bottom panel). The largest discrepancies were seen in the wrist-based Fitbit ${ }^{\circledR}$ during moderate and brisk walking as energy expenditure was overestimated during these stages by $52.4 \%$ and $33.3 \%$, respectively (right wrist).

Inter-device correlations between Fitbit-estimated step counts and energy expenditure of the right hip devices were 0.99 (steps) and 0.96 (energy expenditure), between right and left hip devices were 0.99 (steps) and 0.97 (energy expenditure), and between right and left wrist devices were 0.90 (steps) and 0.95 (energy expenditure).

Our study shows that the Fitbit One ${ }^{\circledR}$ and Fitbit Flex ${ }^{\circledR}$ reasonably and reliably estimate step counts and energy expenditure during walking and running (two of the most common activities among primary care patients); with the hip-based Fitbit One ${ }^{\circledR}$ outperforming the wrist-based Fitbit Flex ${ }^{\circledR}$. With the capability to wirelessly interface with mobile devices and the growing number of platforms/apps that provide patients a means to share health information with their physician, the Fitbit ${ }^{\circledR}$ may be an accurate, reliable, and efficient tool for physicians to track the adoption/maintenance of physical activity programs and support their patient's attempt at an active lifestyle.

\section{Acknowledgments}

Acknowledgement of Grant Support: This work was supported by R01-HL115941 from the National Heart, Lung, and Blood Institute at the National Institutes of Health (NIH; K.W. Davidson) and an NIH Diversity Supplement R01-HL116470-02S1 (K.M. Diaz). 


\section{References}

1. Hebert ET, Caughy MO, Shuval K. Primary care providers' perceptions of physical activity counselling in a clinical setting: a systematic review. Br J Sports Med. 2012; 46(9):625-631. [PubMed: 22711796]

2. VanWormer JJ, Pronk NP, Kroeninger GJ. Clinical counseling for physical activity: translation of a systematic review into care recommendations. Diabetes Spectrum. 2009; 22(1):48-55.

3. Vuori IM, Lavie CJ, Blair SN. Physical activity promotion in the health care system. Mayo Clin Proc. 2013; 88(12):1446-1461. [PubMed: 24290119] 
(A)

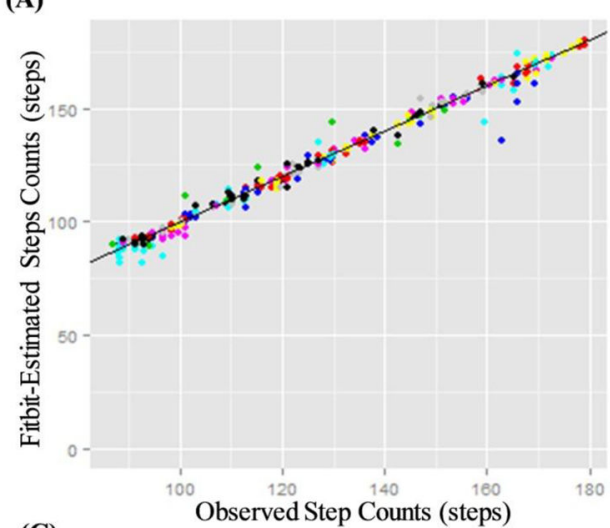

(C)

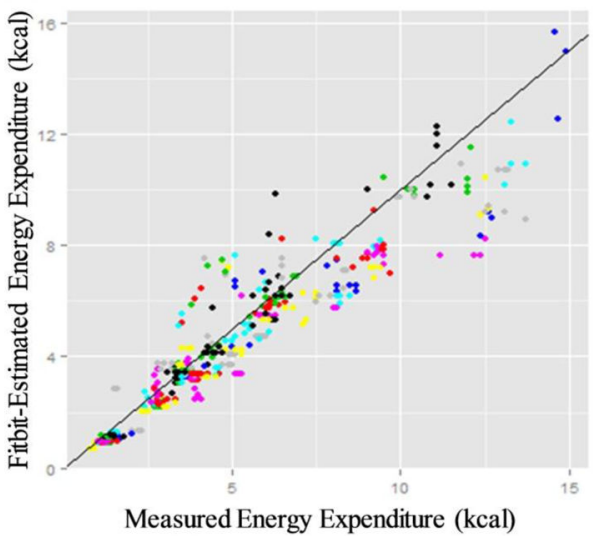

(B)

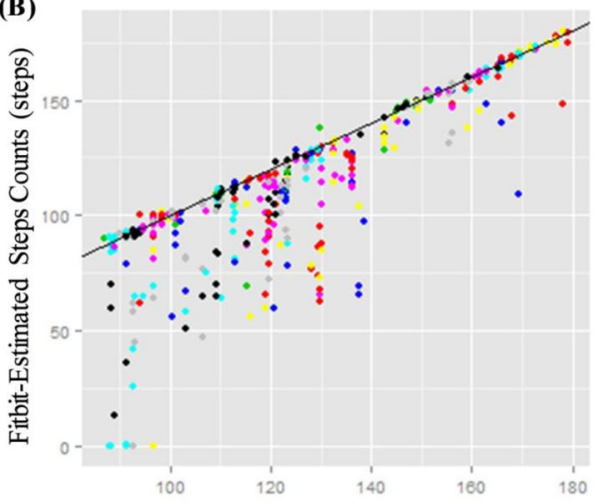

(D) Observed Step Counts (steps)

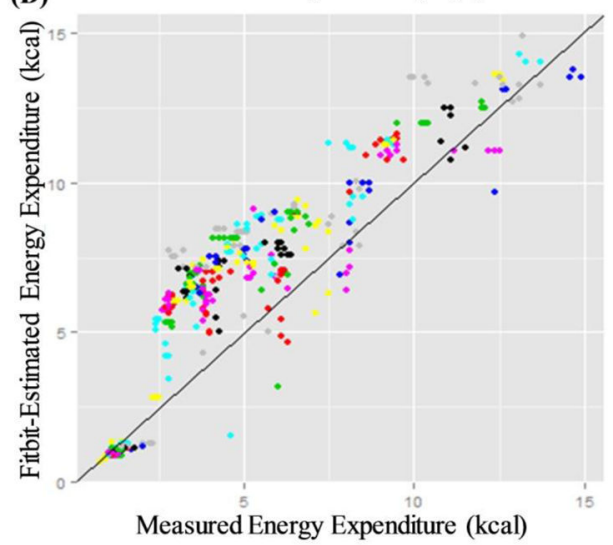

Figure. Relationship of criterion measures of steps counts and energy expenditure counts with Fitbit-estimated step counts and energy expenditure

Top panels: relationship of observed steps counts with hip-based (A) and wrist-based (B) Fitbit-estimated steps counts. Bottom panels: relationship of indirect calorimetry measured energy expenditure with hip-based (C) and wrist-based (D) Fitbit-estimated energy expenditure. 


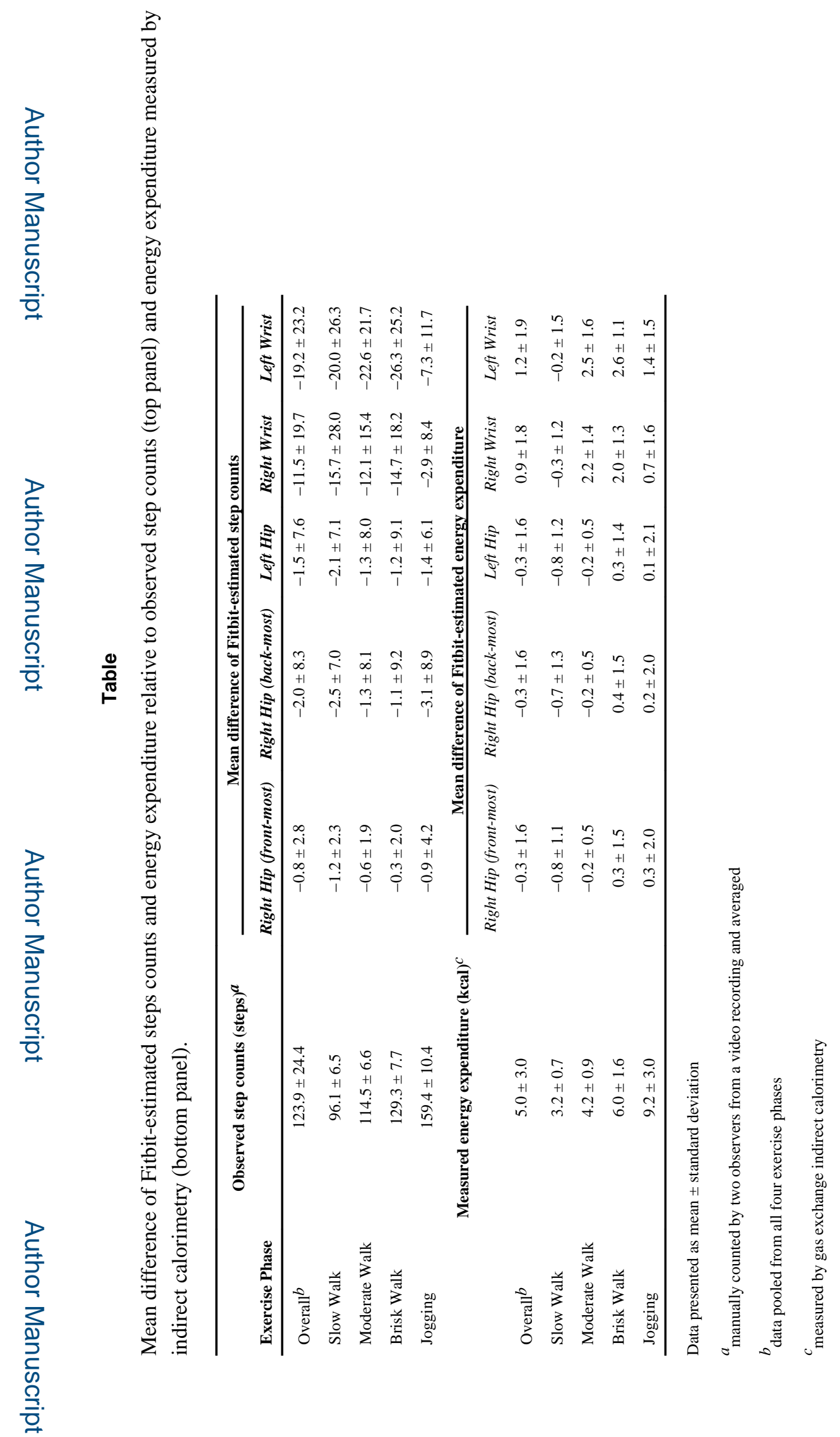

Int J Cardiol. Author manuscript; available in PMC 2016 April 15. 